Full length article

\title{
Network capacity of cognitive radio relay network
}

\author{
Chu-Hsiang Huang, Yen-Chieh Lai, Kwang-Cheng Chen* \\ Institute of Communication Engineering and Department of Electrical Engineering, National Taiwan University, Taipei, 10617, Taiwan, ROC
}

\section{A R T I C L E I N F O}

\section{Keywords:}

Cognitive radio network

Cognitive radio relay network

Cognitive radio

Network coding

Network capacity

Cooperative network

Relay network

\begin{abstract}
A B S T R A C T
After successful dynamic spectrum access, cognitive radio (CR) must be able to relay the message/packets to the destination node by utilizing existing primary system(s) (PS) and/or cooperative/cognitive radio nodes in the cognitive radio network. In this paper, we pioneer the exploration of the fundamental behaviors of interference between CRs and PS in such a relay network via network coding. Interference on PS's network capacity is shown to be unavoidable and unbounded in the one-hop relay network. Extending to the tandem structure, interference is unbounded but avoidable by appropriate constraints. In cooperative relay network, interference is bounded and avoidable. Moreover, parallel cooperative relay network can accommodate more CR transmission pairs. Such an analysis can be generalized to arbitrary networks. We derive that interference is avoidable when at least one route from CR's source to the sink bypasses the bottlenecks of PS. Then under the constraint of no interference to PS, we derive CR's maximum network capacity in such a network. Link allocation to achieve the maximum network capacity can be formulated and solved as a linear programming problem. Consequently, given any network topology, we can determine whether CR's interference is avoidable, and maximize CR's network capacity without interfering PS's network capacity. Simulation results on randomly generated network topologies show that CR's network capacity achieves on average 1.3 times of PS's network capacity with interference avoidance constraint, and demonstrates spectrum efficiency at networking throughput and high availability.
\end{abstract}

(c) 2008 Elsevier B.V. All rights reserved.

\section{Introduction}

Cognitive radio (CR) terminal based on software define radio (SDR) technology [1] has widely drawn attention as a key technology for future wireless communications. CR terminal is a device which can explore the available spectrum to transmit on and can "adapt" communication to connect to various systems. Traditionally, CR aims at utilizing spectrum holes by dynamic spectrum access to enhance spectrum efficiency [11]. With CR terminal's ability to adapt its communication to connect to various

\footnotetext{
th This research is supported by the National Science Council under the project NSC 96-2219-E002-022.

* Corresponding author.

E-mail addresses: b92901138@ntu.edu.tw (C.-H. Huang), b94504014@ntu.edu.tw (Y.-C. Lai), chenkc@cc.ee.ntu.edu.tw (K.-C. Chen).
}

systems, we can form cognitive radio network in which various systems can be connected, and cooperate together $[2,3]$. An important form of general concept of cognitive radio network is that CR terminals must be able to utilize existing primary systems (PS) and/or cooperative/cognitive radio nodes to relay its message. We call this form of network a cognitive radio relay network (CRRN) (Fig. 1). Consequently CRRN is composed of the network of PS and/or cooperative/cognitive radio nodes and the $C R$ terminals utilizing the network to transmit data. We call the source in CRRN other than CR terminals a PS source, and call the network utilized by CR the relay network. Traffic from PS source and CR terminals coexist in the CRRN, hence PS's network capacity may decrease due to CR's interference. We analyze the fundamental behavior of CR's interference to know how to avoid interference to PS when we facilitate CRRN.

Although interference in dynamic spectrum access is well studied [4,5], the fundamental behavior of the 


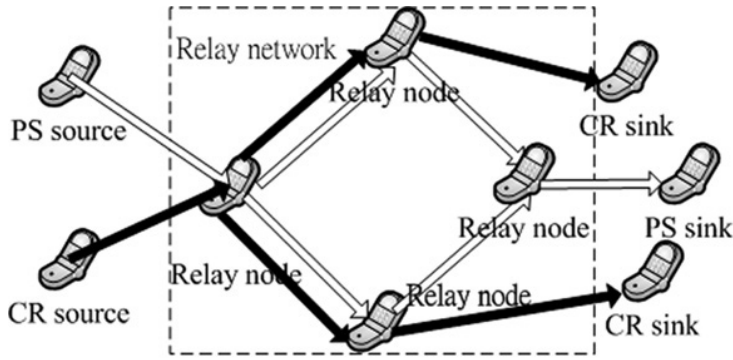

Fig. 1. In cognitive radio relay network, $C R$ utilizes relay network to relay data, hence traffic from CR and PS coexist in the relay network.

interference between CR and PS in CRRN has not yet been studied. By extending the concept in [6], Geng applied network coding to cognitive radio network in [8]. Geng's cognitive radio network is composed of ad hoc CR nodes. However, we utilize network coding to further analyze interference in CRRN, thus we could specify the conditions for CR to avoid interference to PS.

In this paper, we analyze the interference between CR and PS by examining the min-cut capacity variation of PS in CRRN. Min-cut capacity is the capacity of a network, which is derived in the network coding theory [6]. Superposition coding is considered in the analysis because CRRN is likely to adopt Decode-and-Forward (DF) cooperation policy. That is, nodes in relay network decode CR's message and forward it by superimposing CR's message and PS's message. We analyze under the DF policy whether CR's interference is avoidable or bounded in various CRRN topologies. We start our analysis from the simplest network: a one-hop relay network. Then we extend our analysis to the tandem relay network. Next, we analyze parallel structures: cooperative relay network and parallel cooperative relay network. These network topologies are used in cooperative communication with network coding $[9,10]$. The above analysis of bounded and avoidable interference is then generalized to arbitrary CRRN topologies with DF cooperation policy. We aim at avoidable interference analysis due to CR's nature of avoiding interference to PS. First we derive the conditions to enable CR to avoid interference to PS in CRRN. Then we restrict CR and PS to be uni-cast in CRRN to simplify the maximum capacity analysis. Under the constraint of avoiding interference to PS, we derive CR's maximum network capacity, and formulate the link allocation problem to achieve the maximum network capacity as a multi-commodity flow problem. Then we release the constraint on uni-cast, assume PS is multicast and CR is uni-cast, and we show that the problem of link capacity allocation to maximize CR's network capacity in this situation becomes another linear programming problem. Therefore, we can decide whether an arbitrary network can be utilized by CR without interference and maximize CR's network capacity in the CRRN. Finally unavoidable interference and interference bounded condition are also analyzed. Then we conduct simulations on randomly generated CRRN topologies, showing that CR can utilize the PS network to relay without interfering with PS in $92 \%$ of the cases, and is able to get significant network capacity (on average 1.3 times PS's network capacity).

The rest of this paper is organized as follows. The next section introduces the system model and DF cooperation policy (superposition coding) for CRRN. We then analyze the fundamental CRRN topologies in Section 3. Generalized results are provided in Section 4. Simulation result is presented in Section 5. Finally the conclusion is given in Section 6.

\section{System model}

\subsection{Assumptions for our CRRN}

We make the following assumptions to focus our analysis on interference in CRRN and simplify the analysis procedures:

(1) There are one PS source, one CR source, one or a few PS sinks, one or a few CR sinks in the CRRN we analyze.

(2) CR source does not transmit directly to its sinks. That is, we consider only the CR traffic relayed by relay network.

(3) The links in CRRN are uni-directional. Therefore, the networks are able to be modeled as directed graphs.

(4) Building new links between CR nodes and nodes of relay network does not alter the link capacity of other links.

(5) Every edge in the relay network is contained in at least a path from PS source to PS sink. In other words, every link in the relay network should relay the traffic coming from the PS source.

We denote the directed graph of network without CR terminals which utilize the relay network $G=(V, E)$, $V$ is the set of nodes in $G, E$ is the set of links in $G$, and capacity matrix $\mathbf{R}=\left[R_{i j}\right]$ whose entries correspond to link $(i, j) \in E$. Similarly, we denote the directed graph of CRRN $G^{\prime}=\left(V^{\prime}, E^{\prime}\right)$, capacity matrix $\mathbf{R}^{\prime}=\left[R_{i j}^{\prime}\right]$ whose entries correspond to link $(i, j) \in E^{\prime}$. Consequently, the two graphs have following relationships:

1. $V^{\prime}=V \cup s_{C R} \cup t_{C R}, \quad E^{\prime}=E \cup E_{C R-V}$,

2. $R_{i j}^{\prime}=R_{i j} \quad$ if $(i, j) \in E$.

$s_{C R}$ and $t_{C R}$ are the set of CR source and CR sinks correspondingly, $E_{C R-V}$ is the link between CR nodes and relay network nodes. We call $G=(V, E)$ the original network and $G^{\prime}=\left(V^{\prime}, E^{\prime}\right)$ the CRRN in the following sections.

\subsection{Decode-and-Forward cooperation policy: Superposition coding}

Based on the network coding theory model in [6], we define the following components for the network code we consider here:

1. Message set:

$$
\Omega_{P S}=\left\{1, \ldots,\left\lceil 2^{n h_{P S}}\right\rceil\right\}, \quad \Omega_{C R}=\left\{1, \ldots,\left\lceil 2^{n h_{C R}}\right\rceil\right\} .
$$


2. Encoding functions on each link:

For the links which do not relay message from CR:

$$
f_{i j}: \prod_{\left(i^{\prime}, i\right) \in E^{\prime}} A_{i^{\prime} i} \rightarrow A_{i j} .
$$

For the links that relay both CR's and PS's messages:

$$
\begin{aligned}
& f_{i j, P S}: \prod_{\left(i^{\prime}, i\right) \in E^{\prime}} A_{i^{\prime} i, P S} \rightarrow A_{i j, P S} \\
& f_{i j, C R}: \prod_{\left(i^{\prime}, i\right) \in E^{\prime}} A_{i^{\prime} i, C R} \rightarrow A_{i j, C R} .
\end{aligned}
$$

For the links which connect node $i$ to sink:

$$
g_{i}: \prod_{\left(i^{\prime}, i\right) \in E^{\prime}} A_{i^{\prime} i} \rightarrow A_{i} .
$$

3. Decoding in sinks For PS

$$
g_{P S}: \prod_{i} A_{i} \rightarrow \Omega_{P S}
$$

and for CR

$$
g_{C R}: \prod_{i} A_{i} \rightarrow \Omega_{C R} \text {. }
$$

Message sets for PS and CR are $\Omega_{P S}$ and $\Omega_{C R}$. Sources uniformly choose an index from their sets then transmit it on the network. $n$ is the block code length, $h_{P S}$ and $h_{C R}$ are code rates, $f_{i j}$ is the encoding function on link $(i, j)$. In our CRRN, relay nodes decode messages from PS and from CR separately and superimpose them to transmit on the links. So the encoding functions of the links that relay both CR's and PS's messages are $f_{i j, P S}$ and $f_{i j, C R}$, and they separately code CR and PS's messages. Then $A_{i j}=A_{i j, P S} \times A_{i j, C R}$ would be sent on link $(i, j)$. According to these settings, the code rate on each link $(i, j)$ which relays both data from $C R$ and PS is

$R_{i j}=n^{-1} \log _{2}\left|A_{i j}\right|=n^{-1} \log _{2}\left|A_{i j, P S}\right|+n^{-1} \log _{2}\left|A_{i j, C R}\right| .(7)$ This is called superposition coding. This coding scheme superimposes the two network codes together. Although superposition coding sometimes cannot achieve multisource network capacity bounds derived in [7], we use it because it is probably not feasible to jointly compress and code messages from PS and CR. And we do not need to alter PS's network code in CRRN if PS's network capacity is maintained. In other words, in CRRN, PS can use the same network code as if CR terminals do not connect to the network under superposition coding. Superposition coding is a DF cooperation policy.

\subsection{Network capacity under DF cooperation policy}

Under the DF cooperation policy, for those links which contain both traffic from CR and PS, we can denote the capacity of the link $R_{i j}=R_{i j, C R}+R_{i j, P S}$, while $R_{i j, C R}>0$ and $R_{i j, P S}>0$. By the network coding theory, under our settings, we can derive network capacity by calculating the min-cut capacity respective to PS and CR.

We analyze interference by the following procedures. First, we want to derive the network capacity $v$ of original network, $G=(V, E)$ and $\mathbf{R}=\left[R_{i j}\right]$. Then we can derive the network capacity of PS $v_{p}$, the network capacity of CR $v_{C R}$, and the total network capacity $v_{t}=v_{P S}+v_{C R}$ in the CRRN, $G^{\prime}=\left(V^{\prime}, E^{\prime}\right)$ and $\mathbf{R}^{\prime}=\left[R_{i j}^{\prime}\right]$. Thus we are able to know the conditions enabling us to achieve $v_{P S}=v$ and $v_{C R}>0$, which means CR's interference is avoidable. Moreover, we want to maximize $v_{C R}$ and maintain $v_{P S}=v$ by appropriately allocating link capacity. We can also derive whether $v_{P S}$ is always larger than zero under any link capacity allocation. If yes, we call the interference as bounded.

\section{Network capacity analysis on fundamental CRRN topologies}

In different CRRN topologies, CR under the DF cooperative policy may cause interference to PS to different extents. Depending on topology and link capacity, CR's interference can be avoidable and unavoidable, bounded and unbounded. We analyze these interference properties in the following fundamental topologies, and generalize these analyses to arbitrary topologies in the next section. We set the capacity of links between relay nodes and sources or sinks to be infinite. Hence we can focus on the interference on relay network.

\subsection{One-hop relay network and tandem relay network}

We start from the simplest topology analysis: one-hop relay network. There is only one link in this network. Intuitively, as long as CR is transmitting, PS would be interfered by CR's transmission, and not able to transmit if CR occupies full link capacity. Therefore, interference is unavoidable and unbounded in this network.

A tandem relay network is formed by connecting relay nodes in series. The network capacity of such a network is constrained by the smallest link capacity among all links. Hence CR can connect to the network and transmit through those links other than the smallest capacity one, i.e. if $b>a$ in Fig. 2(b). Unless we allocate too much capacity to CR in a link so that the link becomes the smallest capacity link for PS, CR will not interfere with the transmission of PS, hence the interference is avoidable. However, if CR occupies full capacity in any link, PS cannot transmit anything by this relay network. Therefore, CR's interference is unbounded if we do not put any constraint on it.

\subsection{Cooperative relay network}

We form a cooperative relay network by adding a cooperative relay node (node 2 in Fig. 3), thus there are three links and two cuts in the network.

\subsubsection{Network capacity of original network (Fig. 3(a))}

Let the capacity of link $(1,2),(1,3),(2,3)$ be $a, b, c$ correspondingly. The cuts of PS are $\{(1,2),(1,3)\},\{(2,3)$, $(1,3)\}$. Network capacity in this PS network is:

$v=\min (a+b, b+c)$.

\subsubsection{Network capacity analysis of CRRN (Fig. 3(b))}

In cooperative relay network, we connect CR sink to node 2 , the cooperative relay node. Denote the network 

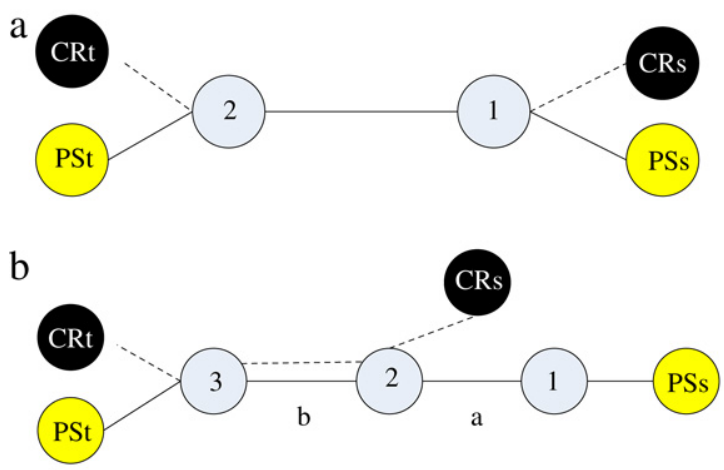

Fig. 2. (a) one-hop relay network (b) tandem relay network. a

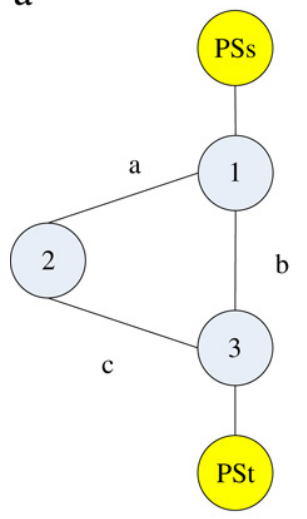

b

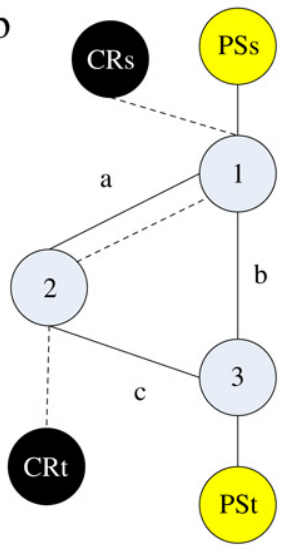

Fig. 3. Cooperative relay CRRN topologies: (a) Original network (b) CRRN: One CR source and one sink.

capacity of CR $v_{C R}$, capacity of PS $v_{P S}$, and the total network capacity of CRRN (CR plus PS) $v_{t}$.

The cut of CR is $(1,2)$. Network capacities of PS and CR are:

$v_{P S}=\min \left(a-v_{C R}+b, b+c\right), \quad v_{C R} \leq a$.

If $a+b>v, v_{C R}$ can be larger than zero while $v_{p}=v$. Under this condition,

$v_{P S}=\left\{\begin{array}{ll}a-v_{C R}+b & \text { if } v_{C R} \geq a-c \\ v & \text { if } v_{C R}<a-c\end{array}, \quad v_{P S}, v_{C R}>0\right.$

$v_{t}= \begin{cases}a+b & \text { if } v_{C R} \geq a-c \\ v+v_{C R} & \text { if } v_{C R}<a-c .\end{cases}$

If we set $v=v_{p}$, we know that

$v_{C R} \leq a-c$.

Hence $a-c$ is the maximum network capacity of CR when $v=v_{P S}$. Note that $v_{C R} \geq 0$, therefore, we always have $v_{P S} \geq b$. This shows that interference of CR to PS is bounded in this case. Under this setting, namely, one CR source and one CR sink, situations are the same when we add them in $(2,3)$, just change $a$ to $c$ in the previous analysis.
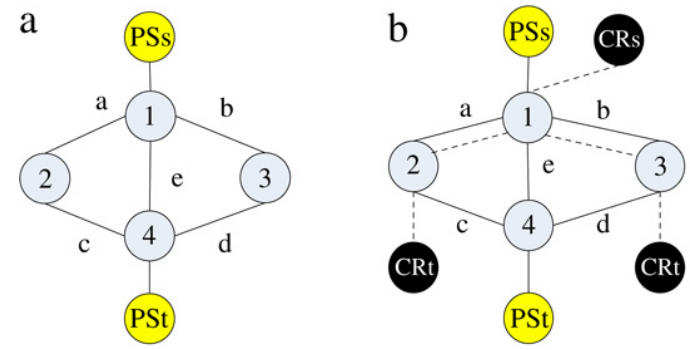

Fig. 4. Parallel cooperative relay CRRN topologies: (a) Original network. (b) CRRN: One CR source and two CR sinks.

From the above analysis, if CR's message is relayed by the links that do not belong to min-cut of PS, then CR's interference to PS is avoidable. Moreover, the interference to PS is bounded in this case. Such observation results from that adding cooperative relay node creates a new route and more cuts, hence CR's interference to PS is avoidable and bounded.

In this case, there are two scenarios of the relationship between CR's and PS's network capacity. When CR's network capacity does not reach its maximum value yet, we can increase CR's network capacity without altering PS's network capacity. After reaching the maximum value of CR's network capacity, network capacities of CR and PS can be allocated in a certain range, but the capacity we add to $C R$ is equal to the capacity we subtract from PS, and vice versa.

\subsection{Parallel cooperative relay network}

So far we have considered only one CR sink. Now we extend our analysis to multiple CR sinks to investigate the interference. In cooperative relay network, we add an extra cooperative relay node (node 2 in Fig. 3), and only one CR sink can be added in under the constraint of avoiding interference to PS. Now we add another node (node 3 in Fig. 4(a)) in to form a cooperative relay structure parallel to the original one. Then we add another CR sink into CRRN (Fig. 4(b)). In this topology, we have four cuts, each with two links.

\subsubsection{Network capacity of original network (Fig. 4(a))}

Let the capacity of link $(1,2),(1,3),(2,4),(3,4),(1,4)$ be $a, b, c, d$, e correspondingly. The cuts of PS are $\{(1,2),(1$, $3),(1,4)\},\{(2,4),(3,4),(1,4)\},\{(1,2),(3,4),(1,4)\},\{(1,3)$, $(2,4),(1,4)\}$. Network capacity of this network is:

$v=e+\min (a+b, c+d, b+c, a+d)$.

\subsubsection{Network capacity analysis of CRRN (Fig. 4(b))}

The cuts of CR are $\{1,2\},\{1,3\}$. Network capacities of PS and CR are:

$$
\begin{aligned}
v_{P S}= & \min \left(a+b-2 v_{C R}, a-v_{C R}+d, b-v_{C R}+c, c+d\right) \\
& +e, \quad v_{C R} \leq \min (a, b) .
\end{aligned}
$$

Therefore, if $a+b, a+d, b+c>v$ that is, $a>c, b>d$, then $v_{C R}$ can be larger than zero while $v_{P S}=v$. 
Under this condition and assuming $a+d>b+c$, we have

$$
v_{P S}=e+ \begin{cases}a+b-2 v_{C R} & \text { if } v_{C R}>b-d, v_{C R}>a-c \\ b-v_{C R}+c & \text { if } v_{C R}>b-d, v_{C R}<a-c \\ c+d & \text { if } v_{C R}<b-d, v_{C R}<a-c\end{cases}
$$

$$
v_{t}=+ \begin{cases}a+b-v_{C R} & \text { if } v_{C R}>b-d \text { and } v_{C R}>a-c \\ b+c & \text { if } a-c>v_{C R}>b-d \\ c+d+v_{C R} & \text { if } v_{C R}<b-d \text { and } v_{C R}<a-c\end{cases}
$$

These cases are symmetric to $a+d<b+c$. If we set $v_{P S}=v$, we have

$v_{C R}=\min (a+d, b+c)-v$.

This topology can accommodate two CR sinks in the two parallel relay nodes, and CR's interference is still avoidable and bounded. We can inference that more CR sinks can exist in parallel cooperative relay CRRN due to the parallel structure created by cooperative relay nodes. However, in two CR sinks and one PS sink case, increasing network capacity of CR may possibly decrease total network capacity. This is due to the two links connecting to two CR sinks belonging to the same min-cut of PS in this case. Therefore if we increase CR's network capacity by $x$, then we decrease PS's network capacity by $2 x$, thus total network capacity is decreased by $x$. But if we appropriately choose the network capacity of $C R$, we still can improve total network capacity and avoid interference to PS.

\section{Generalized results}

From the above analysis, we can observe some crucial relationships between interference properties and network topologies. We list the observations below.

\section{- Avoidable interference}

(1) In one-hop relay network, the interference is always unavoidable because the min-cut includes all the edges in the network

(2) In tandem relay network, if the cut of CR is not included in the min-cut of PS, interference is avoidable.

\section{- Bounded interference}

(3) Interference in cooperative relay network is bounded due to the fact that CR cannot occupy all edges in any cut of PS.

- Multiple CR sinks

(4) In parallel cooperative relay structure with multiple CR sinks, the above properties still hold, but total network capacity in CRRN may decrease when the constraint on avoiding interference to PS is released.

Then we generalize the above observations to arbitrary network topologies under DF cooperation policy. In the following analysis, we denote the network capacity of $\mathrm{CR}$ $v_{C R}$, the network capacity of PS $v_{P S}$, the network capacity of original network $v$, and total network capacity in CRRN $v_{t}=v_{P S}+v_{C R}$. We first elaborate the avoidable interference, then release the constraint on avoiding interference and analyze unavoidable interference and finally derive the condition for bounded interference.

\subsection{Avoidable interference}

In most cases, $\mathrm{CR}$ tries to utilize communication resource under the constraint of avoiding interference to PS. Hence we would emphasize this part and provide most details about how $\mathrm{CR}$ can avoid interference when utilizing PS to relay. Lemma 1 derives the condition for the relay network topologies, and Theorem 1 further derives the condition for CRRN topologies based on Lemma 1. In these network topologies, we derive CR's maximum network capacity in uni-cast PS network in Theorem 2, and then provide algorithms to achieve CR's maximum network capacity in both uni-cast PS and multicast PS. Finally we briefly analyze the total network capacity variation when CR begins interfering with PS.

\subsubsection{Conditions on network topologies}

Lemma 1. Given that every link belongs to at least one mincut in the CRRN. In such CRRN, if $v_{P S}=v$, then $v_{C R}=0$.

Proof. Denote the set of PS cuts $\varphi, \varphi$ contains all the PS cuts whose capacities are equal to $v$. And the capacity of a cut $c$ is denoted $R(c)$ in the original network and $R(c)$ in CRRN. Suppose $\bigcup_{c_{i} \in \varphi} c_{i}=E$. If $v_{C R}>0$, there is at least one link $(i, j)$ such that $R_{i j, C R}>0$. The link capacity of PS on edge $(i, j)$ becomes

$R_{i j, P S}^{\prime}=R_{i j}^{\prime}-R_{i j, C R}=R_{i j}-R_{i j, C R}<R_{i j}$.

This edge $(i, j)$ must belong to some cut $c_{i}$ in $\varphi$. Because $R_{i j, P S}^{\prime}<R_{i j}$, we have $R^{\prime}\left(c_{i}\right)<R\left(c_{i}\right)$. Therefore, we can infer $v_{P S}=R^{\prime}\left(c_{i}\right)<R\left(c_{i}\right)=v$. Hence there is a contradiction. Thus our lemma is proved.

Theorem 1. Set $v_{p}=v$. Then $v_{C R}>0$ if and only if there is no cut contained in the edge set that contains all min-cuts of PS.

Proof. We first prove the "if" part. Let link set $\varphi_{p}=$ $\left\{e_{i} \mid e_{i}\right.$ belongs to any cut whose capacity $\left.=v_{P S}\right\}$, let the set of cuts $\psi_{C R}$ include all cuts of CR, and let $h_{P S}$ be the smallest PS cut capacity among the PS cuts not belonging to $\varphi_{P S}$.

If $c_{i} \not \subset \varphi_{P S}$ for all $c_{i} \in \psi_{C R}$, allocate small capacity $\frac{h_{P S}-v}{m}>0$ to CR in every link $e_{i} \notin \varphi_{p}$. Let $m$ be the number of edges in the relay network. Not more than $m$ edges can belong to the same cut, hence the capacity of every element in $\psi_{C R}$ is larger than or equal to $h_{P S}-m \times \frac{h_{P S}-v}{m}$. Hence $v_{P S}=v, v_{C R} \geq \frac{h_{P S}-v}{m}>0$.

Then we prove the "only if" part. Assume $v_{C R}=\Delta>0$, then the capacity of every element in $\psi_{C R}$ is larger than or equal to $\Delta$. But $v_{P S}=v$, so link capacity for every element in $\varphi_{p}$ allocates no capacity to $\mathrm{CR}$, hence $c_{i} \not \subset \varphi_{P S}$ for all $c_{i} \in \psi_{C R}$.

Note that the "only if" part may not be necessarily true if we consider compress-and-relay cooperative policy instead of DF cooperative relay policy.

4.1.2. CR's maximum network capacity and link capacity allocation algorithms in PS uni-cast network

Then we derive CR's maximum network capacity under the constraint of avoiding interference to PS. To simplify 
the problem, we first consider only one PS sink and one CR sink when we derive CR's maximum network capacity. We define the cut of CRRN to mean the edge set that divides the CRRN into two disconnected networks, one contains CR source and PS source, the other contains CR sink and PS sink. Consequently, a cut of CRRN must contain CR cut and PS cut. In Section 2, we assumed that every edge in the relay network is contained in at least one path from PS source to PS sink. According to this assumption, every cut of CRRN is a cut of PS.

Theorem 2. Let $v_{P S}=v$. CR's maximum network capacity in CRRN is the CRRN's minimum cut capacity minus original network capacity.

Proof. Let CRRN's minimum cut capacity be $v_{C R R N}$. According to [12], we can add a supersource connected to CR source and PS source and a supersink connected to CR sink and PS sink, hence the network capacity of the supersource is $v_{C R R N}$, and $v_{P S}+v_{C R}=v_{C R R N}$. Therefore

$v_{C R}=v_{C R R N}-v_{P S}=v_{C R R N}-v$.

Thus our lemma is proved.

After we derive CR's maximum network capacity, we should find a way to allocate link capacity to achieve CR's maximum network capacity without interfering with PS. The link allocation problem in CRRN can be formulated as a multi-commodity flow problem [13-15]. The messages from CR and PS are the commodities, and CR's maximum network capacity $v_{C R R N}-v$ and PS's network capacity $v$ are the commodities' demands (i.e. flow value). Therefore, we can allocate link capacity to achieve CR's maximum network capacity by solving the multi-commodity flow problem through linear programming [16].

\subsubsection{Link capacity allocation algorithms to achieve CR's} maximum network capacity in PS multicast network

Then we release the assumption of uni-cast, let PS be multicast and CR still uni-cast. For a multicast network, the network capacity is limited by its min-cut capacity. Hence we can formulate the problem of maximum network capacity of $\mathrm{CR}$ by extending the multi-commodity flow problem.

Proposition 1. In CRRN of multicast PS and uni-cast $C R$, the problem of link allocation to achieve maximum network capacity can be formulated as a linear programming problem.

We show Proposition 1 is true in the following. We consider the flow from PS source to each sink separately. Every flow should exceed or at least equal $v$ to guarantee $v=v_{P S}$. Then we choose the maximum flow among the flows to each PS sink on each edge to be the link capacity allocated to PS, and maximize CR's network capacity.

Now we can show that this problem can also be formulated as a linear programming problem. We change the notation of original network capacity from $v$ to $a$ in order to distinguish it from the notation of vertices $v$. Hence our link allocation algorithm in PS multicast network can be formulated as follows. $s_{P S}$ and $s_{C R}$ denote the sources of PS and CR, $t_{P S l}$ and $t_{C R}$ denote the sinks of PS and CR (PS has multiple sinks), $f_{P S i}$ and $f_{C R}$ denote the link capacity (flow) allocated to PS and CR, and $v$ denote the relay nodes.

The linear programming problem of link allocation in PS multicast CRRN is summarized as follows.

Maximize

$\sum_{v \in V_{C R S}} f_{C R}(s, v), \quad V_{C R}=V-\left\{t_{P S l} l=1 \ldots k\right\}$

subject to

(i) $\sum_{v \in V_{i s}} f_{P S i}\left(s_{P S}, v\right) \geq a$

$V_{i s}=V-\left\{s_{C R}, t_{C R}, t_{P S I} \mid l=1 \ldots k\right\}$

(ii) $\sum_{v \in V_{i}} f_{P S i}(u, v)=0 \quad$ where

$V_{i}=V-\left\{t_{C R}, t_{P S \mid} \mid l=1 \ldots k\right.$ except $\left.i\right\}$

$u \in V-\left\{s_{C R}, s_{p}, t_{C R}, t_{P S l} \mid l=1 \ldots k\right\}$

(iii) $\sum_{v \in V_{C R}} f_{C R}(u, v)=0 \quad$ where

$V_{C R}=V-\left\{t_{P S \mid} \mid l=1 \ldots k\right\}$

(iv) $f_{P S i}(u, v)=-f_{P S i}(v, u)$

(v) $f_{C R}(u, v)=-f_{C R}(v, u)$

(vi) $f_{P S i}(u, v) \leq c(u, v)-f_{C R}(u, v)$ for $i=1 \ldots k$.

We can solve the above linear programming by many well-known algorithms such as the simplex algorithm.

The above results provide us with a framework to form the CRRN in which CR can avoid interference to PS. Lemma 1 shows that the original network should have links not on the bottleneck of the network. Min-cut is the bottleneck of a network. Theorem 1 derives the condition that CR should satisfy: min-cuts of PS should not include any cut of CR. That is, we should be able to find at least one route from CR's source to sink that does not go through the bottlenecks of PS. Lemma 1 and Theorem 1 give us a complete characterization of CRRN topology. Given the CRRN's topology and assuming PS and CR are unicast, Theorem 2 gives us CR's maximum network capacity under the constraint of avoiding interference to PS. This is the upper bound of the resource in terms of network capacity that $\mathrm{CR}$ can get by utilizing relay network. Then we formulate the link allocation problem to achieve CR's maximum network capacity as a multi-commodity flow problem, which can be solved by linear programming algorithms. Finally we extend our work to multicast PS and uni-cast CR, showing that the CR maximum capacity link allocation problem is still a linear programming problem hence can be solved accordingly.

\subsubsection{Total network capacity variation corresponding to $C R$ interfering with PS}

We investigate the variation of total network capacity corresponding to the variation of CR's network capacity when CR begins to interfere with PS. By Theorem 2, CR's min-cut must be contained in PS's min-cut which is also CRRN's min-cut when the maximum network capacity is 
achieved. Hence small variation of link capacity allocation without changing the positions of CR's min-cut and PS's min-cut would not change total network capacity. Total network capacity is still CRRN's min-cut capacity. But when we consider that CR is multicast, variation of link capacity allocation may decrease the total network capacity, which is stated in the following lemma.

Lemma 2. If $C R$ is multicast and PS is uni-cast in CRRN, increasing allocation of network capacity to $C R$ decreases total network capacity if the links of different min-cuts of different sinks of CR occupy more than one link belonging to the same min-cut of PS.

Proof. We only prove the case of $2 \mathrm{CR}$ sinks and occupying 2 links belonging to the same min-cut of PS. Other cases are the trivial extensions of this proof. Denote the two links belonging to different CR's min-cuts $e_{1}, e_{2}$, and these two links belong to the same PS min-cut $\varphi_{1}$. Let capacity allocation of link $e_{i}$ be $c_{e i}=c_{e i, P S}+c_{e i, C R}$. Now, if we increase link capacity allocated to CR on $e_{1}$ and $e_{2}$ by $\delta>0$, we have

$c_{e i}=\left(c_{e i}-\delta\right)+\left(c_{e i, C R}+\delta\right)$ for $i=1,2$.

Because $e_{1}$ and $e_{2}$ are on the min-cut of different sinks of CR and belonging to the same min-cut of PS, we have

$v_{C R}^{\prime} \leq v_{C R}+\delta$.

$v_{P S}^{\prime}=v_{P S}-2 \delta$.

$v_{t}^{\prime}=v_{C R}^{\prime}+v_{P S}^{\prime} \leq v_{C R}+v_{P S}-\delta<v_{t}$.

The inequality in (28) is due to that there may be another CR's min-cut other than to which $e_{1}$ and $e_{2}$ belong. By (30), total network capacity of CRRN is decreased by at least $\delta$.

\subsection{Unavoidable interference}

Next we investigate CR's interference to PS when the interference is unavoidable. We restrict our discussion to small capacity allocation to CR, and estimate the bound of capacity interference to PS. The idea and the proof of the following lemma come from the proof of Theorem 1 with some modifications.

Lemma 3. Assume CR's interference is unavoidable. Let link set $\varphi_{p}=\left\{e_{i} \mid e_{i}\right.$ belongs to any cut whose capacity $\left.=v_{P S}\right\}$. There are $m$ cuts of CR contained in $\psi_{P S}$. Then CR's interference to PS's network capacity is bounded by $v_{C R} \times m$, under the constraint that $\psi_{P S}$ is not altered by allocating link capacity to $C R$.

Proof. Assume CR's cuts in $\psi_{P S}$ are not overlapped. We allocate link capacity to CR to achieve some small network capacity $v_{C R}$ under the constraint that $\psi_{P S}$ is not altered. Therefore, PS min-cuts are still contained in original $\psi_{P S}$, and there are $m$ CR's cut getting $v_{C R}$ link capacity in $\varphi_{p}$. Assume these CR's cuts are contained in only one PS mincut, then this cut's capacity will be decreased by $v_{C R} \times m$, and PS's network capacity decreases proportionally. If we release the assumption of non-overlapping CR's cuts or interference on only PS min-cut, interference will be distributed and the effects on network capacity will be decreased. Consequently, the interference will be bounded by $v_{C R} \times m$ under our assumptions.

\subsection{Bounded interference}

Now we investigate the condition for bounded interference generalized from the observations.

Lemma 4. CR's interference to PS is bounded if the edge set which is a union of all CR's paths does not contain any of PS cut.

Proof. We state that CR occupying all the links on all its paths between its source and sinks is the worst case for PS. If the edge set which is a union of all CR's paths does not contain any of PS cut, min-cut capacity of PS is larger than zero even in the worst case. Hence PS's network capacity is always larger than zero. In other words, CR's interference is bounded.

In the networks satisfying the above condition, PS can always maintain its transmission even when CR transgresses the access etiquette.

\section{Simulation results}

In this section, we present the simulation results of our algorithm applied on randomly generated CRRN topologies. We conduct simulations on randomly generated CRRN topologies in a 7 by 7 grid graph, as in an example shown in Fig. 5(a). The relay nodes appear on each grid point on the graph with probability 0.5 . We consider the multicast PS with one source and two sinks, and uni-cast $\mathrm{CR}$, one source and one sink, as shown in Fig. 5(a).

Simulation parameters are set up as follows. The capacity on each link is a randomly selected integer from an interval $(1,3)$. Link exists between two nodes if their distance is smaller than 2 in terms of grid in the graph. Our algorithm executes on 1000 randomly generated CRRN topologies. The histogram of numerical result ratio of CR's maximum network capacity to PS's network capacity is shown in Fig. 6. We use the ratio to present our result to show a comparison between PS's communication resource and CR's communication resource. Ratio -0.5 is defined for CR's maximum network capacity being zero. Simulation results show that CR can utilize PS to relay packets with probability $92 \%$ among randomly generated CRRN topologies. We also can observe that the cases in which $\mathrm{CR}$ can get network capacity equal to or with small difference with PS's network capacity occur most frequently. And on average, CR's network capacity is 1.3 times of PS's network capacity to enhance spectrum efficiency at networking throughput. By this result, we are able to know approximately the probability distribution of CR's maximum network capacity when it utilizes PS network to relay.

Investigating in the CRRN topologies in which $\mathrm{CR}$ acquires higher network capacity, we can observe that either PS's network capacity is constrained by one or a few small min-cuts (Fig. 5(b)) or the distance between CR's source and sink happens to be small (as in an example in Fig. 5(c), CR only needs to go through one or two hops inside the relay network to reach its sink as marked in the network). We can intuitively infer these characteristics from our theoretical results. If the number of PS min-cuts is small and their capacities are small, PS can use only a small 

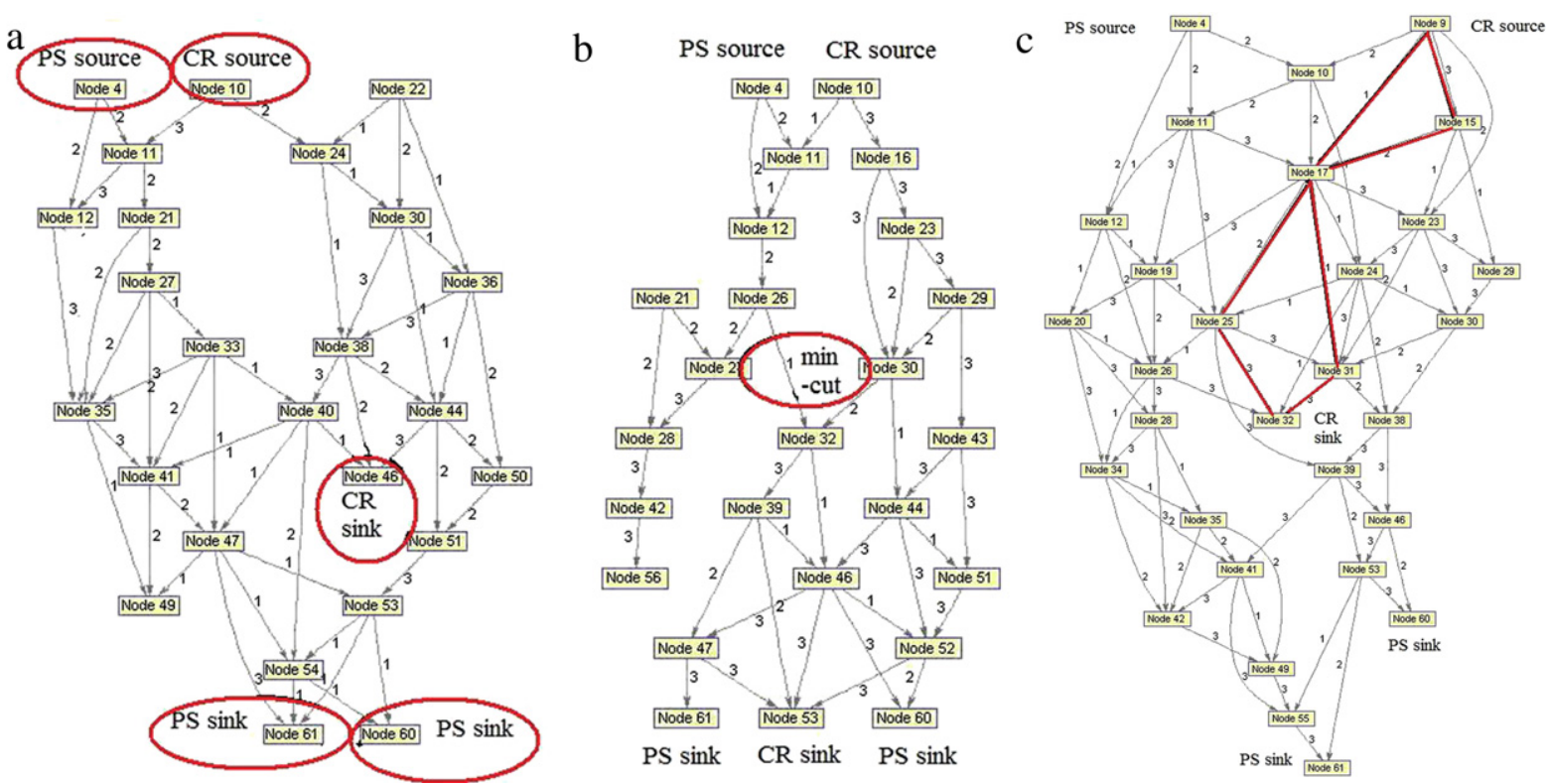

Fig. 5. (a) Example of randomly generated CRRN network topologies (b) An example for PS's network capacity being constrained by a small min-cut (c) An example of small distance $\mathrm{CR}$ (one or two hops inside relay network between source and sink in this case).

Histogram of the ratio of CR's network capacity to PS'snetwork capacity

Relative Frequency (\%)

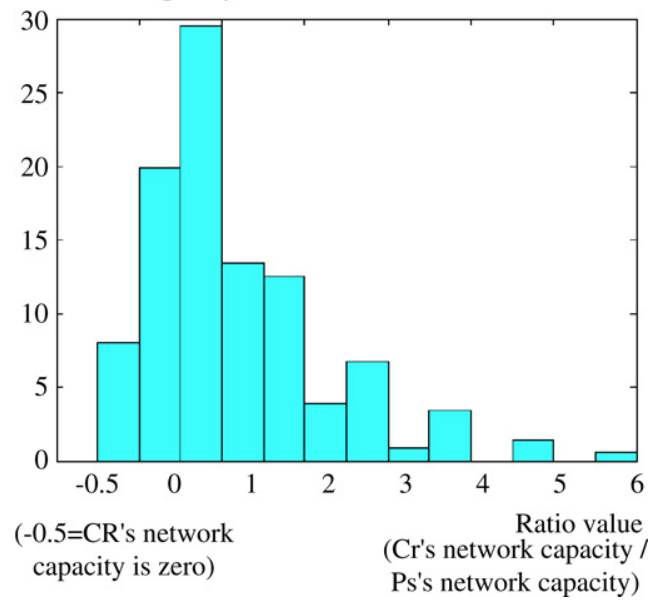

Fig. 6. The histogram of the ratio of CR's network capacity to PS's network capacity.

amount of resource in the network. Consequently, CR has more opportunities to utilize such resource. On the other hand, if CR utilizes fewer hops in CRRN, it is able to utilize more resource in those hops if such hops are not on the bottlenecks of PS. In these situations, we can allocate more capacity to CR without interfering with PS. CR may have maximum network capacity zero because of the condition stated in Theorem 1 or there is no route existing between CR source and sink.

By our simulation results, we know that $\mathrm{CR}$ can successfully utilize PS network to relay with significant network capacity gain in most of the randomly generated CRRN topologies.

\section{Conclusion}

In this paper, we analyze the interference characteristics corresponding to network topologies when CR utilizes PS to relay in CRRN. When CR utilizes PS to relay, we adapt the DF cooperative policy. We start from fundamental network topologies to see whether CR's interference is avoidable and bounded. We derive that interference is unavoidable in one-hop relay, avoidable but unbounded in tandem network, and bounded in cooperative relay structure. Then we extend this to the multiple CR sink case in parallel cooperative relay network. When generalizing the theoretical results, we put emphasis on avoidable analysis due to CR's nature of avoiding interference to PS. First we derive the generalized condition on CRRN topologies for CR's interference to be avoidable: min-cuts of PS should not include any cut of CR. Hence we should have at least one route of CR that bypasses the bottlenecks of PS when designing CRRN. In these kind of topologies, we derive CR's maximum network capacity in PS uni-cast CRRN under the constraint of avoiding interference to PS. We also show that link capacity allocation to achieve maximum network capacity can be formulated as a linear programming problem in both uni-cast and multicast PS. When CR transcends interference constraint, we describe total network capacity variation corresponding to CR's network capacity variation. We then analyze unavoidable interference and estimate the bound of interference in a small CR network capacity range. Finally, we derive the generalized condition on CRRN topologies for CR's interference to be bounded. We simulate the randomly generated CRRN topologies and execute our link allocation algorithm to derive CR's maximum network capacity. On average, CR's maximum network capacity is 1.3 times PS's network capacity. By our analysis on interference characteristics in CRRN, 
routing and scheduling can be developed to enhance network efficiency of applying CRRN. Therefore, CRs using PS to cooperatively relay packets are shown to achieve spectrum efficiency at networking throughput and at high availability.

\section{References}

[1] J. Mitola, G.Q. Maguire, Cognitive radio: Making software radios more personal, IEEE Personal Communications 6 (4) (1999) 13-18.

[2] Kwang-Cheng Chen, Yu-Jeng Peng, Neeli Prasad, Ying-Chang Liang, Sumei Sun, Cognitive radio network architecture: Part I - General structure, in: ACM ICUIMC, Seoul, 2008.

[3] D. Raychaudhuri, et al., CogNet - An architecture for experimental cognitive radio networks within the future internet, in: Proc. of MobiArch 2006, pp. 11-16.

[4] Zhao Qing, B.M. Sadler, A survey of dynamic spectrum access, Signal Processing Magazine, IEEE 24 (3) (2007) 79-89.

[5] Yiping Xing, Chetan N. Mathur, M.A. Haleem, R. Chandramouli, K.P. Subbalakshmi, Dynamic spectrum access with QoS and interference temperature constraints, Mobile Computing, IEEE Transactions 6 (4) (2007) 423-433.

[6] R. Ahlswede, Cai Ning, S.-Y.R. Li, R.W. Yeung, Network information flow, IEEE Transactions on Information Theory 46 (4) (2000) 1204-1216.

[7] Song Lihua, R.W. Yeung, Cai Ning, Zero-error network coding for acyclic networks, IEEE Transactions on Information Theory 49 (12) (2003) 3129-3139.

[8] Lijuan Geng, Ying-Chang Liang, Francois Chin, Network coding for wireless ad hoc cognitive radio networks, in: Personal, Indoor and Mobile Radio Communications, PIMRC 2007, IEEE 18th International Symposium on Sept. 2007, pp.1-5, 3-7.

[9] Xiao Lei, T. Fuja, J. Kliewer, D. Costello, A network coding approach to cooperative diversity, IEEE Transactions on Information Theory 53 (10) (2007) 3714-3722

[10] Shengli Fu, Kejie Lu, Qian Yi, Murali Varanasi, Cooperative network coding for wireless ad-hoc networks, in: Global Telecommunications Conference, GLOBECOM'07, 26-30 Nov. 2007, IEEE, 2007, pp. 812-816.

[11] J. Mitola III, Cognitive radio for flexible mobile multimedia communications, in: Mobile Multimedia Communications, 1999. (MoMuC'99) 1999 IEEE International Workshop on 1999, pp.3-10.

[12] L.R. Ford Jr., D.R. Fulkerson, Flows in Networks, Princeton Univ.Press, 1962.

[13] T.C. Hu, Multicommodity network flows, Operations Research 11 (1963) 344-360.

[14] R.E. Gomory, T.C. Hu, Synthesis of a communication network, J. SIAM 12 (1964) 348-369.

[15] Refael Hassin, On multicommodity flows in planar graphs, Networks 14 (2) (1984) 225-235.

[16] Thomax H. Cormen, Charles E. Leiserson, Ronald L. riverst, Clifford Stein, Introduction to Algorithms, The MIT Press, 2001, pp. 788-789.

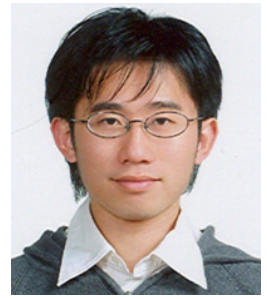

Chu-Hsiang Huang received B.S. in electrical engineering from the National Taiwan University in 2007, and is now working toward M.S. in the Institute of Communication Engineering, National Taiwan University. His research interests are cognitive radio networks and sensor networks.

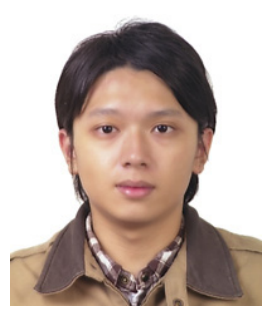

Yen-Chieh Lai is working toward his B.S. in electrical engineering at the National Taiwan University. His current interests are in cognitive radio networks and theory of wireless networks.

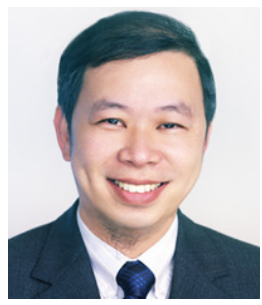

Kwang-Cheng Chen received B.S. from the National Taiwan University in 1983, M.S. and Ph.D. from the University of Maryland, College Park, United States, in 1987 and 1989, all in electrical engineering. From 1987 to 1998, Dr. Chen worked with SSE, COMSAT, and IBM Thomas J. Watson Research Center, National Tsing Hua University in mobile communication networks and related research. Dr. Chen is the Distin guished Professor and Irwin T. Ho Chair Professor at the Institute of Communication Engineering and Department of Electrical Engineering, National Taiwan University, Taipei, Taiwan, ROC. He hold several visiting positions with Technical University of Delft in Netherlands, HP Labs. in US, Aalborg University in Denmark. Dr. Chen actively involves the technical organization of numerous leading IEEE conferences, including 1996 IEEE International Symposium on Personal Indoor Mobile Radio Communications, IEEE Globecom 2002, 2007 IEEE Mobile WiMAX Symposium, and IEEE Vehicular Technology Conference Spring 2010. He has served editorship with many prestigious international journals: IEEE Transaction on Communications, IEEE Communications Letters, IEEE Communication Surveys, IEEE Wireless Communications Magazine, International Journal of Wireless Information Networks, IEEE Journal on Selected Area in Communications, ACM/Blatzer Journal on Wireless Networks, Wireless Personal Communications, and Frontier of Communications and Information Theory, etc. Dr. Chen has authored and coauthored over 200 technical papers and 18 US patents. Dr. Chen is an IEEE Fellow, and recipient of many awards and honors. Dr. Chen's research interests include wireless communications and wireless networks, cognitive and nano-communications. 